\title{
FACE LOCALIZATION AND DETECTION BASED ON SYMMETRY DETECTION AND TEXTURE FEATURES
}

\author{
Chuen-Horng Lin \\ National Taichung University of Science and Technology \\ No. 129, Sec. 3, Sanmin Rd., Central Dist., Taichung City 404, Taiwan \\ linch@nutc.edu.tw
}

Jyun-An Cai

National Taichung University of Science and Technology

No. 129, Sec. 3, Sanmin Rd., Central Dist., Taichung City 404, Taiwan impossible_0623@hotmail.com

Shik-Kuan Liao

National Taichung University of Science and Technology

No. 129, Sec. 3, Sanmin Rd., Central Dist., Taichung City 404, Taiwan

skliao@nutc.edu.tw

\section{ABSTRACT}

This study refers mainly to the characteristics of symmetry and texture features in order to correctly locate a face within an image. Since we target facial expression and illumination variation in a facial image, this first requires an equalization process of adaptive smoothing of the shadows of the face caused by varying illumination. Following this, for symmetry axis detection, the study will address: Gradient Detection, Image Width and Location of Symmetry Axes, Symmetry Axes for Gradient Histogram (SAGH) and Selection; Weight is also added to strengthen symmetry characteristics. In order to verify the accuracy of the method, this study will use 6 experimental methods, namely SAGH, SAPG, WSAGH, WSAPG, WSAGH for no adaptive smoothing, and WSAPG for no adaptive smoothing. The image database used for this experiment is the Yale Face Database, with facial images that are subjected to different illumination, masked by shelters and displaying varying facial expressions. The experiment results show that the WSAPG method is the most accurate; achieving a $96.36 \%$ LM value, with the lowest GM value; it was the most successful at locating a face in the image. Hopefully it will be applied to enhancing current face recognition technology. 
Keywords: Face Detection, Adaptive Smoothing, Symmetry Detection, Texture Feature

\section{INTRODUCTION}

Face recognition technology is part of the Biometric Systems field, and was used in the early stages of digital identity and crime detection. With the evolution of technology, face recognition technology has become widely used in other areas, like the popular social network, Facebook, where face recognition technology identifies the possible location of a face, and allows the user to label the person in the picture, the user will then be able to know where in the picture that person is. Face recognition technology can be applied to video ${ }^{1}$ : if face recognition technology is applied to video, and integrated with the drivers' records, it can assist the police in crime detection by identifying the face locations of people violating the law.

Photography and photo equipment are widely applied in all aspects of life today, assisting people by making up for insufficiencies in visual management, and also producing huge amounts of people image data. A means of instantly identifying each person in such images is a very important subject for future visual images. Face recognition compares the similarity of facial characteristics, and identification of the correct characteristic range is necessary for identifying facial characteristics; thus the location of a face plays a very important role in identifying facial characteristics.

Face recognition technology mainly uses facial characteristics to achieve recognition. The difference between face recognition technology and other Biometric Systems is that it does not require special equipment in order to acquire facial characteristics for recognition; thus, it is suitable for easy use by the general public. For this reason, many researchers are focusing on face recognition and related studies. Current face recognition technology study uses color or gray images to conduct experiments. Color images use facial skin characteristics to conduct recognition ${ }^{2}$, while gray images cannot use these characteristics, using only facial characteristics to conduct recognition. Current recognition methods using facial characteristics include: the template matching $\operatorname{method}^{3}$, feature extraction ${ }^{4}$, 5 , line edge $\operatorname{map}^{6}$, etc. When conducting face recognition, the face is usually located first before conducting recognition, in order to avoid noise interfering with the result. In the past, complicated calculations were 
involved in locating the face, such as ${ }^{7}$ using statistics of Principle Component Analysis (PCA) to project the facial characteristics in high-dimensional space, and selecting the important characteristics. High calculation cost is required if there are several characteristics involved; this study utilizes the symmetry relation of a face to identify the symmetry axis and complete location.

In past studies, researchers have aimed to determine the symmetrical relationship and identify the symmetry axis. The information of the symmetry axis was used to achieve the purposes of the experiments. Like the searching method of symmetry axis by C. Sun et al., these studies mainly applied a gradient to acquire the direction of the object, used a histogram to statistically summarize the gradient direction, and finally used the Fourier method to identify the symmetry axis ${ }^{8}$. In current experiments, for the most part, the symmetrical relationship of the object is used, as proposed by P. C. Hsieh et al. A mirror image is generated in accordance with the symmetry relationship of the face, and face illumination examined ${ }^{9}$. The current study's aim is to find the symmetry axis of the face image, and to use the symmetry axis to locate the face for face recognition. The primary method of this study also uses the symmetry relationship of the face to locate it, hoping that face location can be identified using the symmetry axis for face recognition, thus avoiding manual cutting or complicated calculations for determining face location.
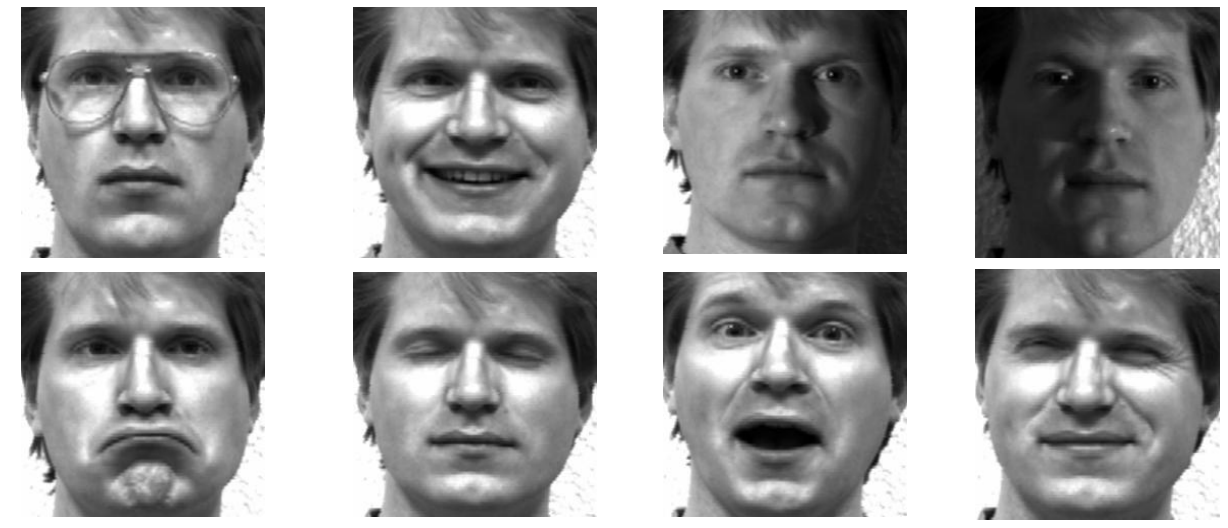

Figure 1. The face image under variations of illumination, expression and occlusion

In an image, there are often different facial expressions, and the image is often affected by varying illumination, as shown in Figure 1, which makes locating the face difficult, and creates fault areas of facial characteristics, rendering face recognition inaccurate. This study, therefore, first uses the left-right symmetrical relationship of the face to 
determine and locate the face area, detect the symmetry axis of the face, and then apply texture features to the face characteristics in order to identify and locate the important face area.

\section{PROPOSED METHOD}

This study provides a face detection method for images exposed to different illumination and depicting different facial expressions, enabling the face detection program to correctly locate the face in the image. First, we detect the shadow of the image and remove it. Owing to the symmetrical relationship of the human face, this study conducts symmetry detection after removing the shadow to find the symmetry of the face. Finally, the face is located using the symmetry axis. This study has adopted adaptive smoothing, symmetry detection and face localization steps to complete face location; the processing procedure is shown in Figure2.

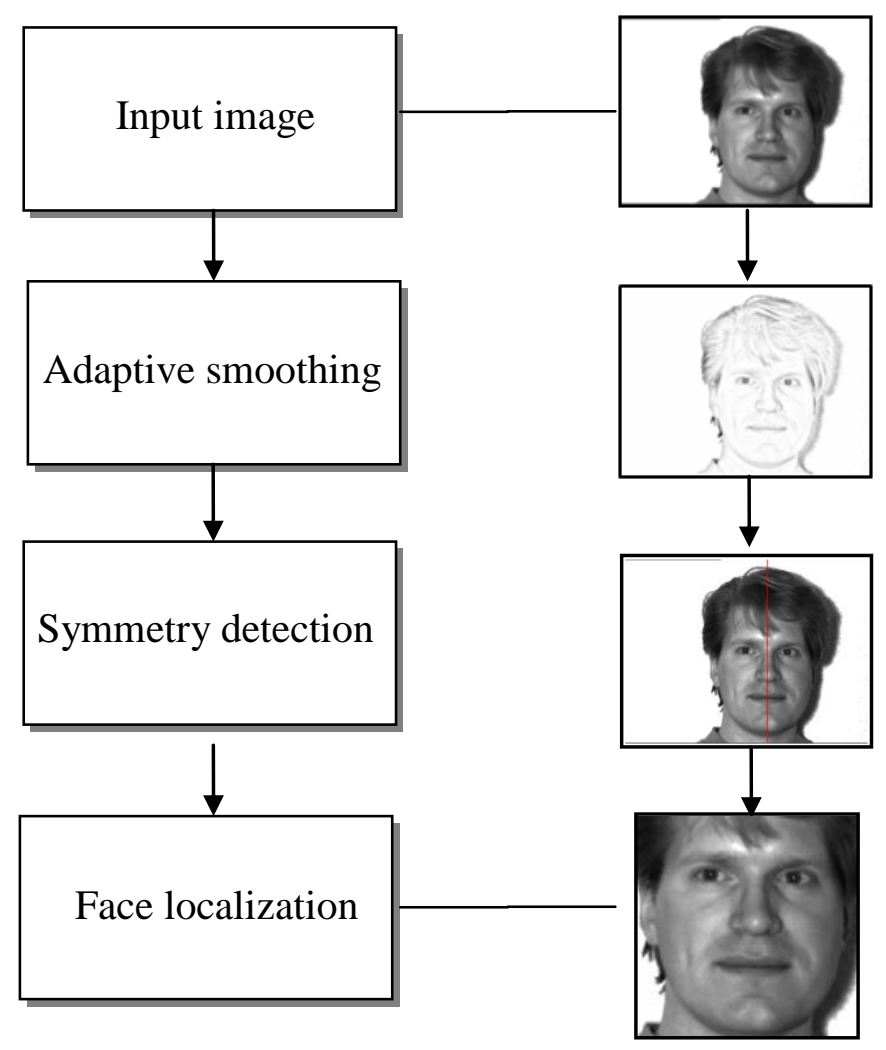

Figure 2. The flow of face method 


\subsection{Adaptive Smoothing}

Images exposed to various illuminations are shown in Figure 3. Owing to different illumination size and direction, different sizes and shapes of image are formed on the face, which severely affects the detection of face. This section will describe the effective processing of shadow in the image, for face location.
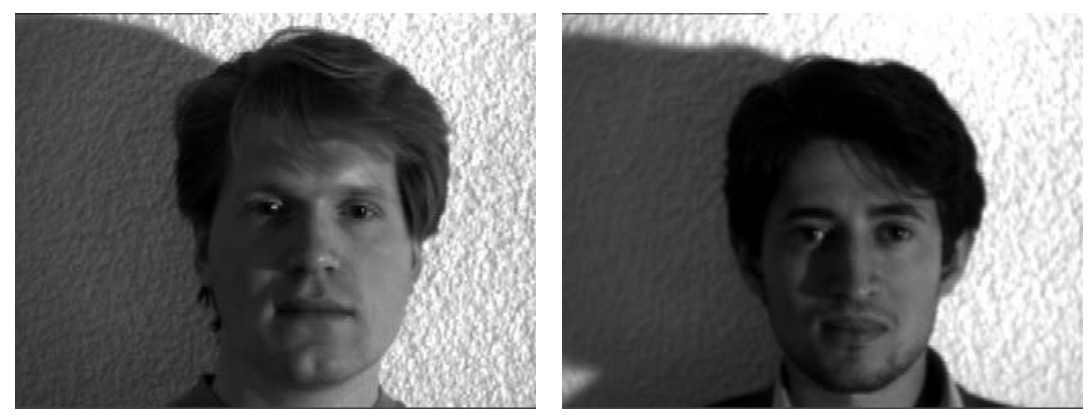

Figure 3. The face image under variation of illumination

In past studies, the equalization process was adopted in order to process images with different lighting and direction ${ }^{10,11}$. This paper adopts the easier and quicker method of adaptive smoothing ${ }^{10}$. This method selects a new image gray level from the biggest value of the original image pixel (already processed by a $3 \times 3$ smoothing kernel) and the original gray level. For the purpose of deleting the shadow of the image, iterative image processing will be used after the image is processed. Iteratively processing the image for $t$ times will produce the coordinators $(x, y)$ of $t+1$, and its intensity, $I^{(t+1)}(x, y)$, will be shown as the following ${ }^{10}$ :

$$
I^{(t+1)}(x, y)=\max \left\{\tilde{I}^{(t)}(x, y), I^{(t)}(x, y)\right\}
$$

The value of $\widetilde{I}^{(t)}(x, y)$ is $I^{(t)}(x, y)$ processed by a $3 \times 3$ smoothing kernel can be shown as follows:

$$
\tilde{I}^{(t)}(x, y)=\frac{1}{N(x, y)} \sum_{j=-1}^{1} \sum_{i=-1}^{1} I^{(t)}(x+i, y+i) \times w(x, y)(i+1, j+1),
$$

where $w(x, y)$ represents the smoothing kernel on the coordinate $(x, y)$ and $N(x, y)$ is the aggregation of the region of $w(x, y)$. The smoothing kernel is the differential value of gray level of an image's pixel with the 
neighboring image's pixel. If the differential value is smaller than $T(x, y)$, it should be a smoothing region; if not, a non-smoothing region.

For the smoothing kernel for each pixel of an image, the sum of absolute difference $\Delta^{(0)}(x+i, y+j)$ between the pixel $(x, y)$ and neighboring pixels of the original image $I^{(0)}$ is defined as follows:

$$
w(x, y)(i+1, j+1)=\left\{\begin{array}{cc}
1, & \text { if } \Delta^{(0)}(x+i, y+j)<T(x, y) \\
0, & \text { otherwise }
\end{array}\right.
$$

where

$$
\Delta^{(0)}(x+i, y+j)=\left|I^{(0)}(x, y)-I^{(0)}(x+i, y+j)\right|,
$$

and

$$
T(x, y)=\alpha \sum_{i=-1}^{1} \sum_{j=-1}^{1}\left|I^{(0)}(x, y)-I^{(0)}(x+i, y+j)\right| .
$$

The $\alpha$ is set to equal 0.5 in this paper. A shadowed image is used in this paper, and the experimental image processed after adaptive smoothing is shown in Figure 4.

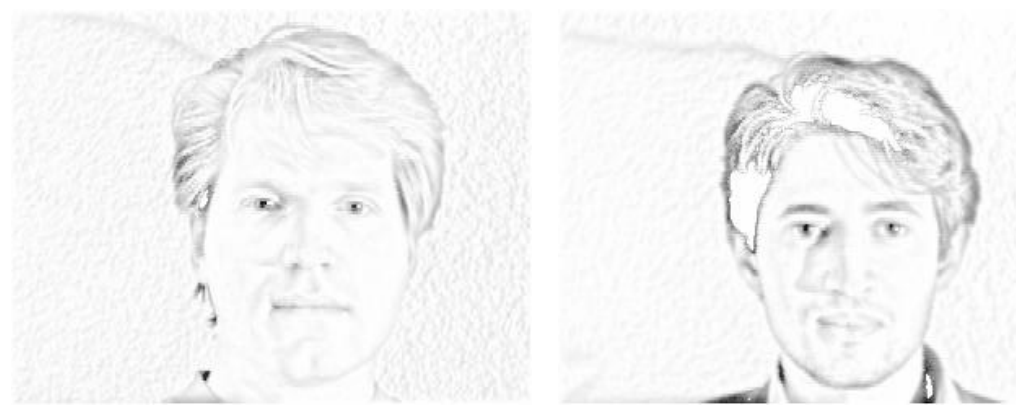

Figure 4. The results of adaptive smoothing

\subsection{Symmetry Detection}

This section provides an introduction to the processing of detection using the symmetry axis. The primary purpose is to symmetrically detect the human face through the symmetry of its left and right sides, thereby obtaining its location in the image. Because of some characteristics of the eyes, eyebrows, nose, mouth and ears, the left and right sides of human 
face have a very obvious symmetry. This paper takes the texture feature as the basis for calculating the symmetry of a human face.

\subsubsection{Gradient Detection}

First, we take the image already processed by adaptive smoothing as $\hat{I}$, and the image in which noise is deleted through a Gaussian filter as $f$. Next, Sobel edge detection is employed to detect the gradient feature. The gradient magnitude $\nabla f$ and direction $\theta(x, y)$ for $f(x, y)$ of pixel at coordinates $(x, y)$ are given by:

$$
\nabla f=g(x, y)=\sqrt{f_{x}^{2}(x, y)+f_{y}^{2}(x, y)} \text { and } \theta(x, y)=\tan ^{-1}\left(\frac{f_{y}(x, y)}{f_{x}(x, y)}\right),
$$

where $f_{x}$ is the edge variation in the horizontal direction and $f_{y}$ is the same in the vertical direction. In this paper a $3 \times 3$ block is used to compute the variations in the horizontal and vertical directions $f_{x}$ and $f_{y}$. A gradient image can be created with gray levels $0 \sim 255$, as shown in Figure 5 .
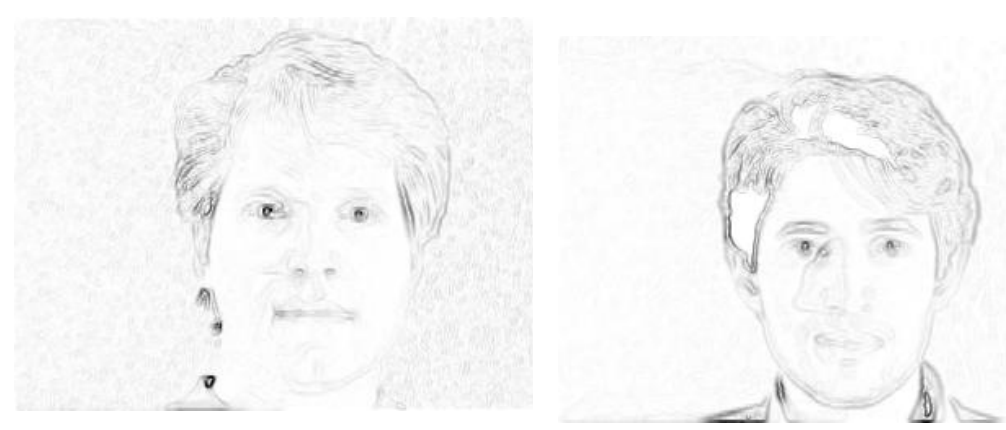

Figure 5. Gradient image

\subsubsection{Image Width and Location of Symmetry Axes}

The symmetry axis represents the direction of the vertical line of the height ( $x$ direction) and width ( $y$ direction) shown in Figure 6 . The range of the image width is 0 to $W$. The image width information for the gradient in the height direction of an image can be used for symmetry detection. This paper provides two ways of measuring symmetry axes using a gradient image: the Symmetry Axis for Gradient Histogram (SAGH) and the Symmetry Axis for Pixel Gradient (SAPG). 


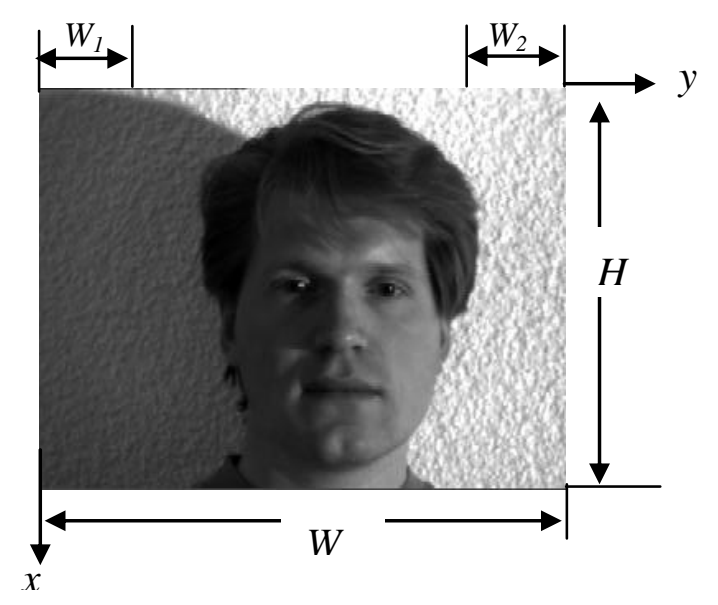

Figure 6. The variables of an image

\subsubsection{Symmetry Axes for Gradient Histogram (SAGH)}

This paper uses the gradient of an image to determine its symmetry axis. It takes one axis at random, and computes the aggregation through the gradient values of the left and right sides. The maximum value will be called the symmetry axis. Firstly, the gradient of every pixel on the $x$ direction will be added up to form bins, and the Gradient Histogram will be drawn using the $y$ direction, which is shown in Figure 7. The formula is as follows:

$$
h(y)=\sum_{i=0}^{H} g_{i}
$$

where $g_{i}$ is the gradient value of the $i$-th pixel.

In this instance, the whole human face is inside the image, and is not shown on the edge of the image. Therefore, the range $y$ of the image width is from $W_{1}$ to $W_{2}$, as shown in Figure 6. We used a gradient image at location $y$ of the histogram with length $\delta$ from the left and $\delta$ from the right, as shown in Figure 7, to calculate the following function:

$$
H(y)=\sum_{\delta=1}^{W_{2}-W_{1}} h(y+\delta) h(y-\delta),
$$


where $H(x)$, the maximum value computed by the formula, represents the symmetry axis of the direction of the width of the image, shown as follows:

$$
S_{S A G H}=\arg \max \left(H(y), y=W_{1}, \ldots, W_{2}\right) .
$$

The above $S_{S A G H}$ is the symmetry axis computed by SAGH in this paper.

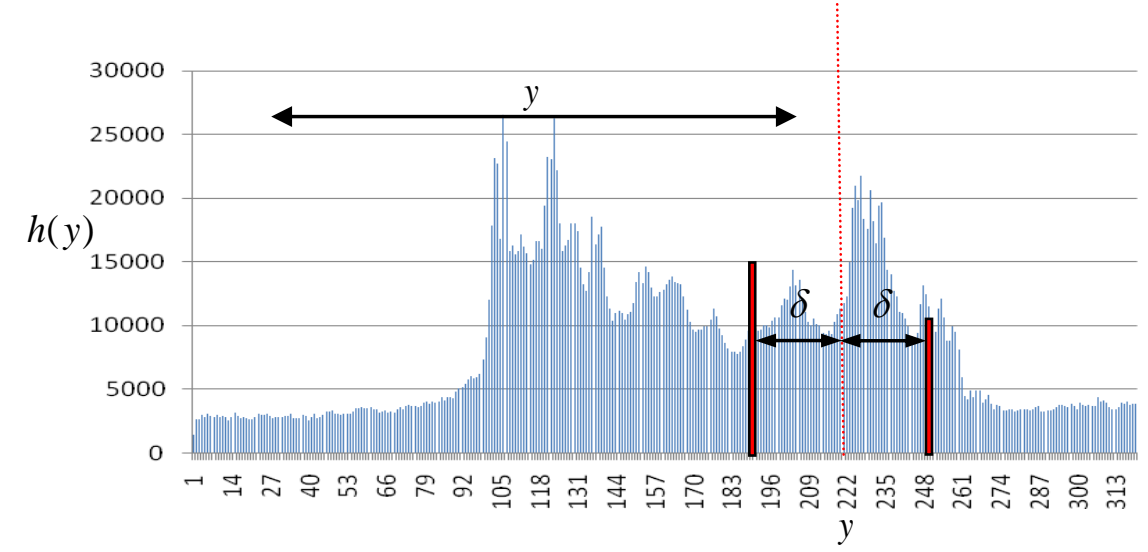

Figure 7. Gradient histogram of an image

\subsubsection{Symmetry Axis for Pixel Gradient (SAPG)}

SAGH, the basis of symmetry, is mainly the aggregation of the gradient in the $x$ direction, and can be speedily calculated. The characteristics of symmetry will be created in the $y$ direction, but will not necessarily be created in the $x$ direction. Therefore, this paper proposes a method in which the gradient of a pixel determines the symmetry axis of the image.

SAPG randomly selects one axis, computes the product of the gradient of the pixel point of the left and right sides and aggregates all the products on this axis. We call the axis on the largest value the symmetry axis. First, randomly select $y$ as the would-be symmetry axis and then use this axis to time the corresponding gradient value of the left and right pixels; the result is shown in Figure 8. Finally, the aggregation of the products is shown as follows:

$$
G(y)=\sum_{\delta=1}^{W_{\mathrm{v}}-W_{1}} \sum_{x=0}^{H-1} g(x, y+\delta) g(x, y-\delta),
$$


where $g(x, y)$ is the gradient magnitude of the pixel at coordinates $(x, y)$. Select the maximum value from the values $G(y)$ of the would-be symmetry axis and define it as the symmetry axis. The formula is shown as follows:

$$
S_{S A P G}=\arg \max \left(G(y), y=W_{1}, \ldots, W_{2}\right) .
$$

The above $S_{S A P G}$ is the symmetry axis computed by the SAPG of this paper.
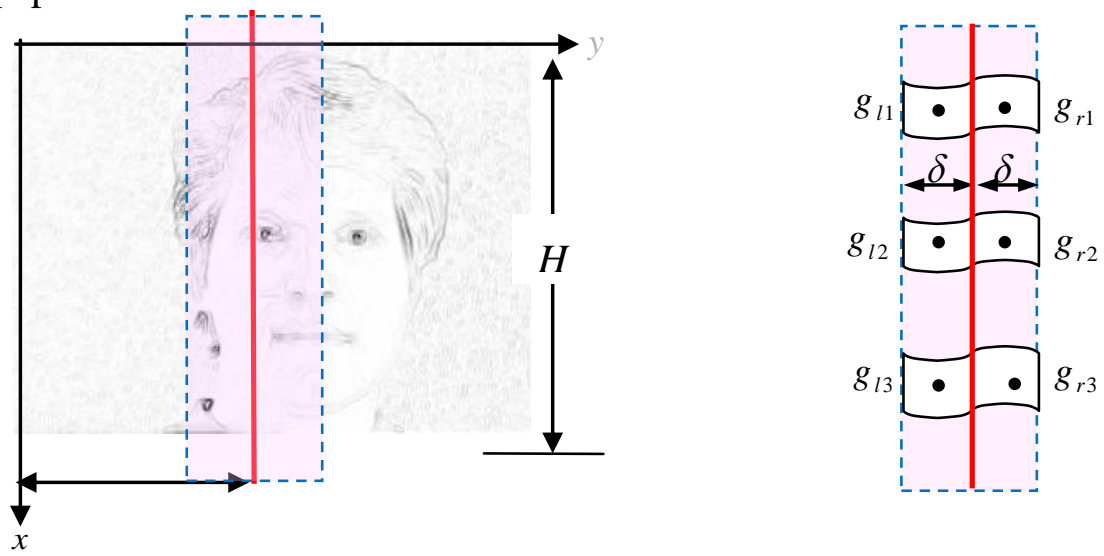

Figure 8. The variables of a gradient image

\subsubsection{Selecting $W_{1}$ and $W_{2}$}

Because the symmetry axis of the human face does not appear on the edge of the image, this paper hypothesizes that the range of the symmetry axis is from $W_{1}$ to $W_{2}$. In order to select the appropriate range of the symmetry axis without increasing the calculating time, this paper adds up every pixel of the $x$ direction of the original image as bins, and draws a histogram using the $y$ direction. We then calculate the average value of the histogram $h(y)$ and compare $h(y)$ and $m_{h}$ in that order, starting from $y=0$ to the right. The formula is shown as follows:

$$
m_{h}=\frac{1}{W} \sum_{y=0}^{W-1} h(y) .
$$

The first time the location $h(y) \geq m_{h}$ is determined, we define it as $W_{1}=y$, shown in Figure 9. Then, compare $h(y)$ and $m_{h}$ in order, 
starting from $y=W-1$ to the left. We define the location where $h(y) \geq$ $m_{h}$ is first determined as $W_{2}=y$.

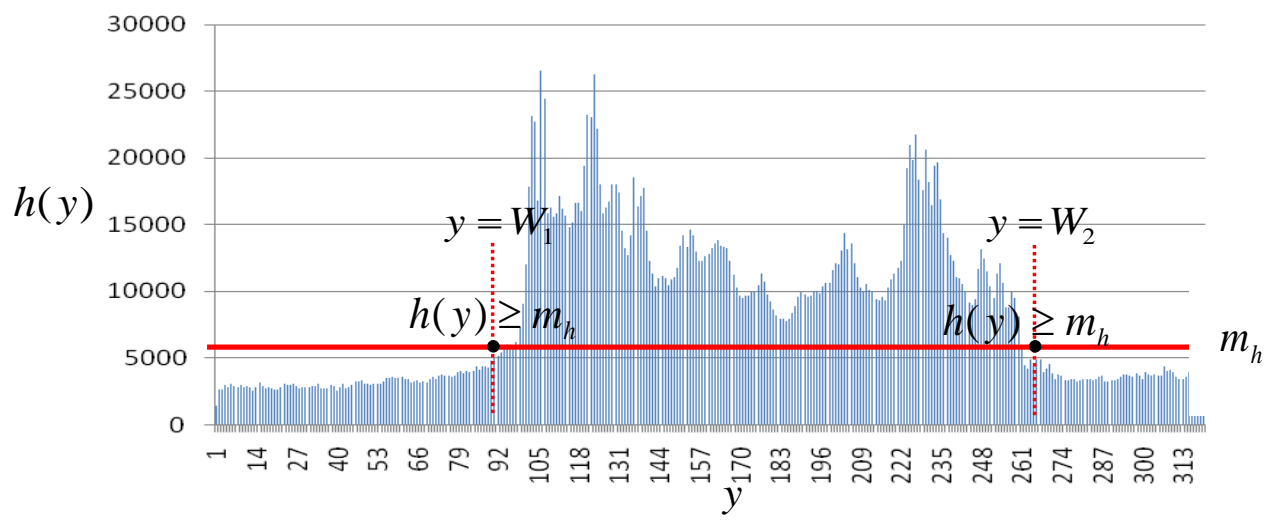

Figure 9. The variables of the gradient histogram

\subsection{Weight of SAGH and SAPG}

SAGH and SAPG, aiming at the corresponding left and right side of any axis, select the symmetry axis based on the bins and the product of gradient values on pixels. By the definition of symmetry, the corresponding values should be equal; however, the algorithms of SAGH and SAPG are modified in this paper. Therefore, this paper proposes to take the rate of the differential value as the weight, and briefly represents Weight of SAGH and Weight of SAPG as WSAGH and WSAPG. The formula (7) of WSAGH can be rewritten as follows:

$$
H(y)=\sum_{\delta=1}^{W_{2}-W_{1}} w(y, \delta)[h(y+\delta) h(y-\delta)]
$$

where $\quad w(y, \delta)=1-\frac{|h(y+\delta)-h(y-\delta)|}{|h(y+\delta)+h(y-\delta)|}$, and the selection of the symmetry axis is as follows:

$$
S_{W S A G H}=\arg \max \left(H(y), y=W_{1}, \ldots, W_{2}\right) .
$$

The formula (9) will be rewritten as follows to represent WSAGH:

$$
G(y)=\sum_{\delta=1}^{W_{\mathrm{v}}-W_{1}} \sum_{x=0}^{H-1} w(x, y, \delta)[g(x, y+\delta) g(x, y-\delta)],
$$


where $w(x, y, \delta)=1-\frac{|g(x, y+\delta)-g(x, y-\delta)|}{|g(x, y+\delta)+g(x, y-\delta)|}$, and the selection of the symmetry axis is as follows:

$$
S_{W S A P G}=\arg \max \left(G(y), y=W_{1}, \ldots, W_{2}\right) .
$$

\subsection{Localization and Detection}

The location of a human face in this paper serves to show the range wherein the characteristics of a human face can be expressed. This area will be the area for the identification of a human face in the future. This area is called the location of a human face. The characteristics of a human face are the shape of the face, eyebrows, eyes, nose, mouth, etc. For this reason, this paper selects a pixel block $(120 \times 140)$ which matches these characteristics, and takes the symmetry axis selected above as the central axis for searching from the top to the bottom, as shown in Figure 10. We search for the most complicated texture in the area, and consider that as the correct location of the face. The most complicated texture in this paper means the aggregation value of the gradient in this area. The larger the value is, the more complicated the feature will be, and the more likely it is that this is the central part of the human face. If the value is less, the feature will be less complicated.

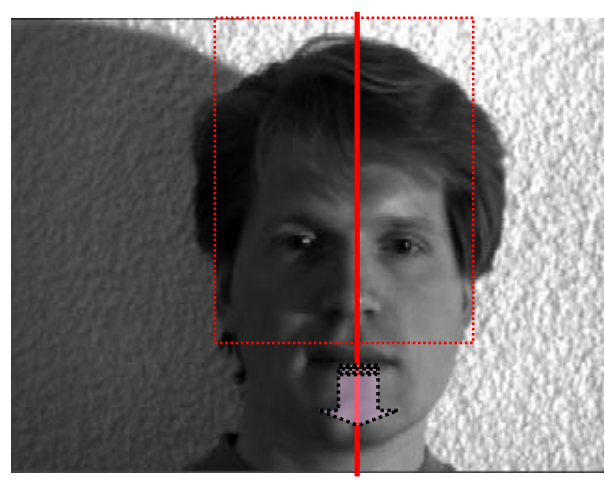

$S$

Figure 10. The location of human face

\section{EXPERIMENTAL RESULTS}

In order to prove the effectiveness and accuracy of the proposed method in this study, we used the Yale Face Database ${ }^{12}$ for our experiments. The database contains images of faces, not only with different expressions and lighting, but also with occlusion (glasses). In this 
study, we carried out an experiment which calculated the locations of the symmetry axis and locations of human faces, respectively. The software used for the experiment was Matlab 9.0. The following paragraph will introduce the experimental image database, our evaluation method for this experiment, and the results of the experiment.

\subsection{The Image Database}

The Yale image database was divided into two parts: the Yale Face Database and the Yale Face B Database. The experiment in this study mainly used the Yale Face Database shown in Figure 11. There are 165 images in total in the database of this experiment. Each image was $320 \times$ 243 in size. Images were composed of 15 individuals, every individual had 11 facial expressions with different illuminations, and some faces had occlusion.

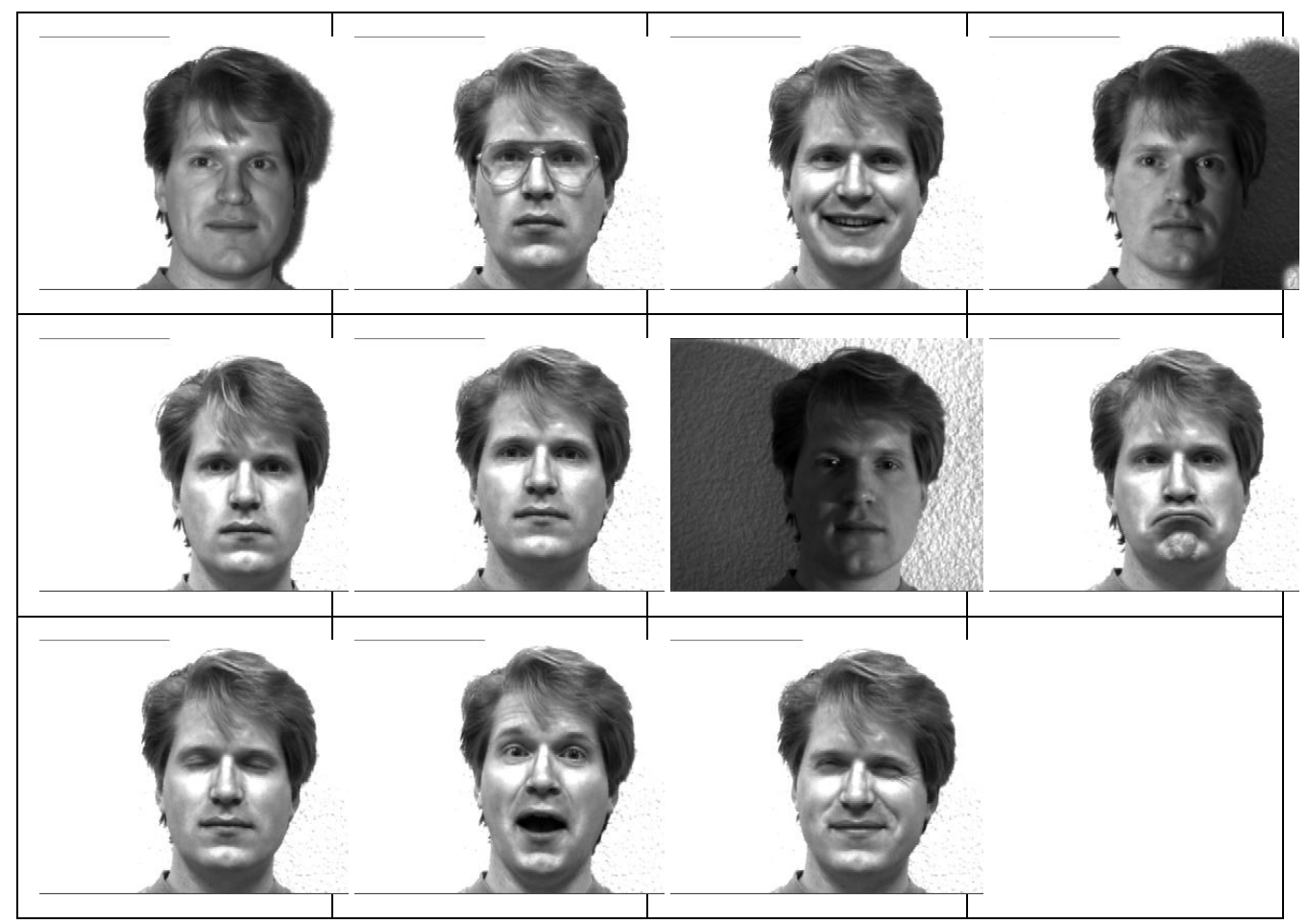

Figure 11. Yale face database

\subsection{Performance Measurement}

The assessment methods of the experiment results are as follows. First, the symmetry axes of human faces were artificially marked with a visual marker, and then used as the Ground truth of the images. Next, the 
Ground truth was used as an evaluation criterion. Two major methods were used to evaluate the symmetry axis: Local Measurement (LM) and Global Measurement (GM).

The LM evaluation method took every experimental image for evaluation and gave an evaluation result (True or False) to each image. The assessment criterion was in accordance with the width (W) of the original image, as shown in Figure 12. The derived symmetry axis value was then compared with the Ground truth value. If the difference between them was in $\mathrm{T}$ range, the result was true, otherwise, false. LM can be expressed as follows:

$$
L M=\frac{1}{N} \sum_{i=1}^{N} P
$$

where

$$
P=\left\{\begin{array}{cc}
1, & \text { if } S-G T \leq T \\
0, & \text { otherwise }
\end{array}, \text { where } \pm 1 \%<T<5 \%,\right.
$$

where $G T$ denotes the symmetry axes of the Ground truth, $F_{T}$ is the symmetry axis of the experimental system, and $N$ is the total number of images in the database. In other words, a higher $L M$ value means a better consistency between the symmetry axes from the experimental system and the Ground truth.
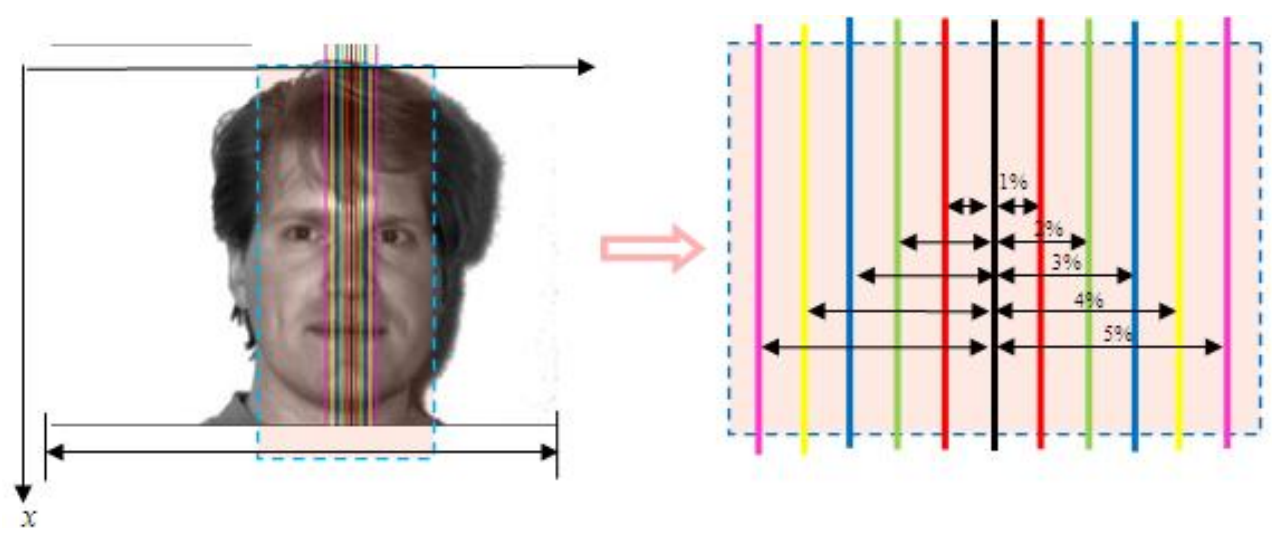

Figure 12. The variables of performance measurement

The $G M$ evaluation method in this study used the derived symmetry axis of all experimental images to calculate the difference with the Ground truth of each image, called the error value. The experimental error values 
of each image were then totaled and finally averaged. $G M$ can be defined as follows:

$$
G M=\frac{1}{N} \sum_{i=1}^{N} S_{i}-G T_{i}
$$

In other words, a smaller GM indicates better symmetry axis precision.

\subsection{Experimental Results}

This experiment used the Yale Face Database mentioned above, and the experimental methods were SAGH and SAPG. Of these methods, SAGH takes bins of the histogram as the basis for the symmetry axis, while SAPG is based on the gradient of pixels. To demonstrate the characteristics of their symmetry more clearly, we also added weights individually to the methods, then denoted as WSAGH and WSAPG. Moreover, these two methods used adaptive smoothing and non-adaptive smoothing to analyze and compare, respectively. The results of this paper are given in Table 1. The experimental results show that the $L M$ of SAPG is higher than SAGH, WSAPG is higher than SAPG, and WSAPG is higher than SAPG, for non-adaptive smoothing. In particular, when the difference between the value of the symmetry axis and the Ground truth is less than 5\%,LM values derived by WSAPG are up to $96.36 \%$. Better results are presented in bold font. Clearly the WSAPG method has achieved a better $G M$ in various images than any of the other five methods have.

Table 1. Comparison of $L M(\%)$ and $G M$

\begin{tabular}{ccccccc} 
$\pm 0.1 \%$ & $31.52 \%$ & $38.79 \%$ & $73.33 \%$ & $76.36 \%$ & $56.36 \%$ & $61.21 \%$ \\
$\pm 0.2 \%$ & $52.12 \%$ & $56.36 \%$ & $81.82 \%$ & $83.64 \%$ & $73.94 \%$ & $77.58 \%$ \\
$\mathrm{LM}(\%) \pm 0.3 \%$ & $67.88 \%$ & $70.91 \%$ & $90.30 \%$ & $92.73 \%$ & $83.03 \%$ & $86.67 \%$ \\
$\pm 0.4 \%$ & $76.97 \%$ & $78.79 \%$ & $93.94 \%$ & $95.15 \%$ & $85.45 \%$ & $89.09 \%$ \\
$\pm 0.5 \%$ & $88.48 \%$ & $88.48 \%$ & $95.15 \%$ & $96.36 \%$ & $87.27 \%$ & $90.30 \%$ \\
\hline $\mathrm{GM}$ & 8.9939 & 8.2848 & 4.0000 & 3.4121 & 9.9818 & 8.6788 \\
\hline
\end{tabular}

This paper chose an image for illustration (as shown in Figure 13), and the symmetry axes derived from the six methods are illustrated with 
red lines. From the results, we can see that WSAPG determined the symmetry axis more accurately than the other five methods did.

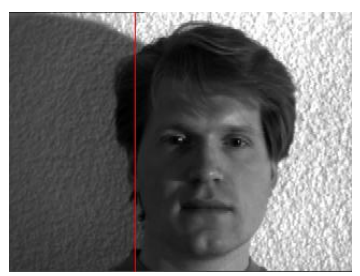

SAGH

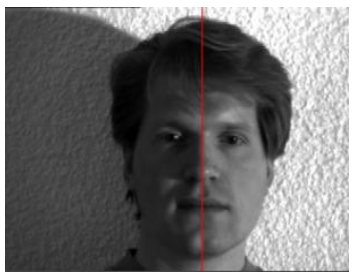

WSAPG

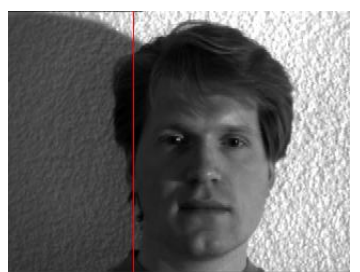

WSAGH

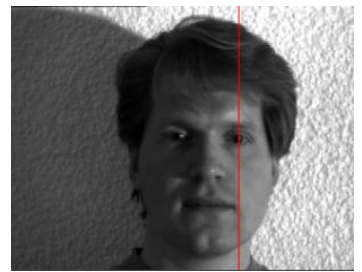

SAPG for nonadaptive smoothing

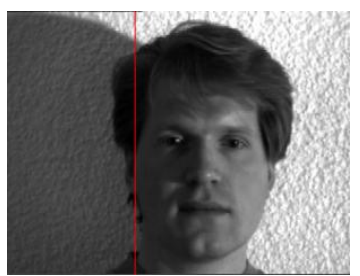

SAPG

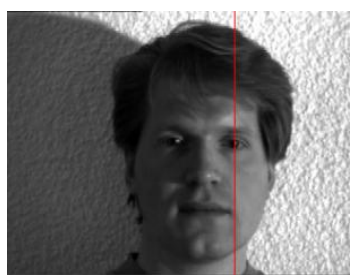

WSAPG for nonadaptive smoothing

Figure 13. Comparison of the symmetry axes results

Because the WSAPG method had the highest accuracy, it was chosen to be used in the experiment with the Yale Face Database with a variety of illuminations and partial shields on human faces. These included: face images with central lighting, with/without glasses, happy face, left lighting, normal expression, right lighting, sad face, sleepy face, surprised face and blinking expression, respectively (from left to right, from top to bottom). The results are shown in Figure 14. The experimental results show that in this paper WSAPG can find symmetry axes very accurately for the faces under different illuminations and faces with shelter and different expressions, except for one image, which got a slightly deviated result.

Finally, we used the WSAPG method for an experiment on localization and detection. First, we used WSAPG to find the axes of symmetry of the faces, and then localized the more important parts of the faces. The result is shown in Figure 15. We used the whole Yale Face Database for this experiment, in which we can see 8 different faces subjected to different illumination and with different facial expressions. We can see that the proposed WSAPG can successfully localize the facial location. 


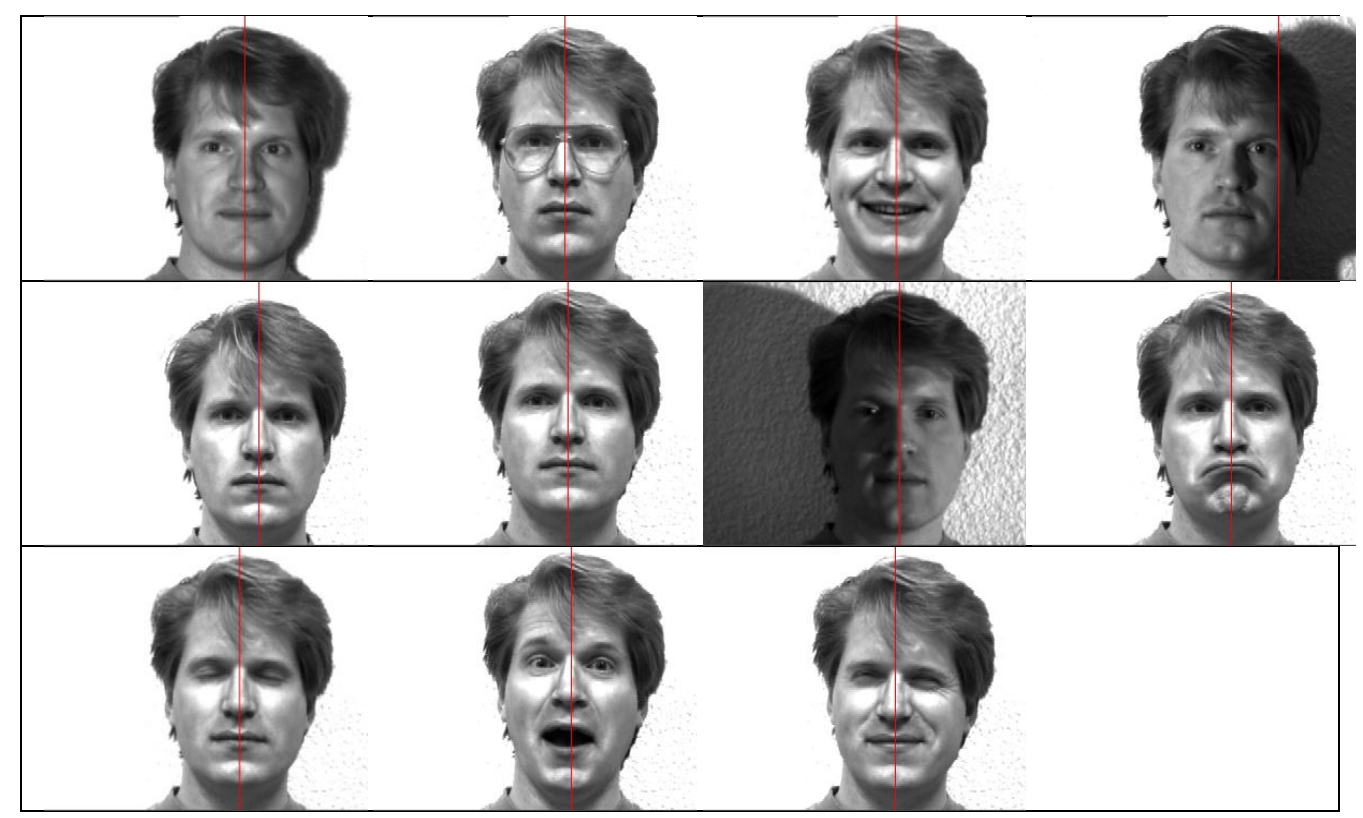

Figure 14. The symmetry axes results of WSAPG

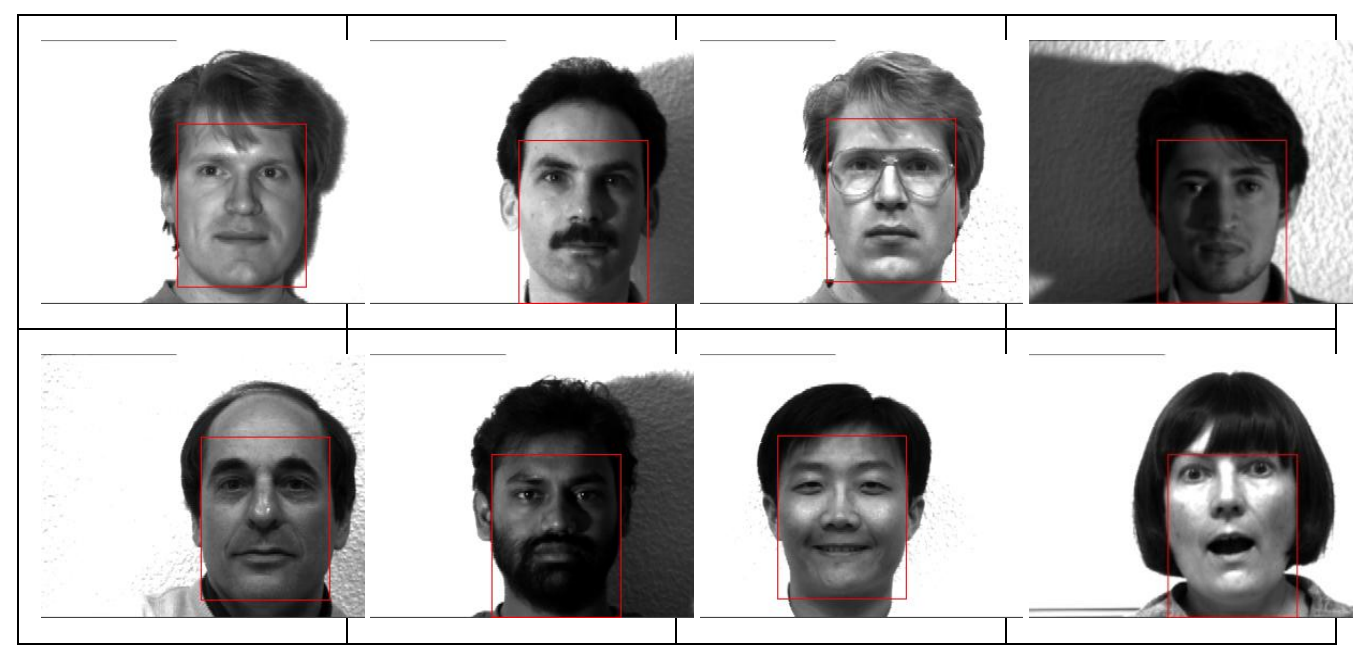

Figure 15. Faces localization

\section{CONCLUSIONS}

The proposed method aimed first to detect the axis of symmetry of a face, and then to localize the important facial area, according to the axis of symmetry. We proposed SAGH, SAPG, WSAGH, WSAPG and WSAGH for non-adaptive smoothing and WSAPG for no adaptive smoothing, according to different procedures and methods. The experimental subject 
is the Yale Face Database, which consists of facial images subjected to different illumination, masked by shields and with various facial expressions. As for the experimental results, the WSAPG method performed better than the other five methods did. Moreover, the received LM value was as high as $96.36 \%$, and the GM value of the WSAPG method, the smallest. The WSAPG method was not affected by varying illumination, masking shelters or varying facial expressions, and can precisely find the axis of symmetry. Furthermore, it can successfully localize the face. Therefore, it is the proposed method since it can localize the important part of face quickly and precisely. We believe that this approach will be a valuable contribution to the future improvement of face recognition technology.

\section{REFERENCES}

[1] J.U. Yun, H.-J. Lee, and A.K. Paul, Face detection for video summary using lamination-compensation and morphological processing. Pattern Recognition letters, 30(9), p856-860, 2009. doi:10.1016/j.patrec.2009.04.010.

[2] Z. Jin, Z. Lou, J. Yang, and Q. Sun, Face detection using template matching and skin-color information. Neurocomputing, 70(4-6), p794-800, 2007. doi:10.1016/j.neucom.2006.10.043.

[3] Y.C. Feng, P.C. Yuen, and A.K. Jain, A hybrid approach for generating secure and discriminating face template. IEEE transactions on information forensics and security, 5(1), p103-117, 2010. doi:10.1109/TIFS.2009.2038760.

[4] D.H. Kim, S.U. Jung, and M.J. Chung, Extension of cascaded simple feature based face detection to facial expression recognition. Pattern Recognition letters, 29(11), p1621-1631, 2008. doi:10.1016/j.patrec.2008.04.006.

[5] W. Zhang, Z. Lin, and X. Tang, Tensor linear Laplacian discrimination (TLLD) for feature extraction. Pattern Recognition, 42(9), p1941-1948, 2009. doi:10.1016/j.patcog.2009.01.010.

[6] Y. Gao, and M.K.H. Leung, Face recognition using line edge map. IEEE transactions on pattern analysis and machine intelligence, 24(6), p 764-779, 2002.

[7] W.S. Yambor, B.A. Draper, and J.R. Beveridge, Analyzing PCA-based face recognition algorithms: Eigenvector selection and distance measures. In H. Christensen and J. Phillips (Eds.), Empirical Evaluation Methods in Computer Vision (p39-60), Singapore: World Scientific Press, 2002. doi:10.1142/9789812777423_0003. 
[8] C. Sun, and D. Si, Fast reflection symmetry detection using orientation histograms. Real-Time Imaging, 5(1), p 63-74, 1999. doi:10.1006/rtim.1998.0135.

[9] P.C. Hsieh, and P.C. Tung, Shadow compensation based on facial symmetry and image average for robust face recognition. Neurocomputing, $\quad$ 73(13-15), $\quad$ p2708-2717, 2010. doi:10.1016/j.neucom.2010.04.015.

[10] Y.K. Park, and J.K. Kim, Fast adaptive smoothing based on LBP for robust face recognition. Electronics Letters, 43(24), p1350-1351, 2007. doi:10.1049/el:20071809.

[11] Z.-U. Rahman, D.J. Jobson, and G.A. Woodell, Investigating the relationship between image enhancement and image compression in the context of the multi-scale retinex. Journal of Visual Communication and Image Representation, 22(3), p237-250, 2011. doi:10.1016/j.jvcir.2010.12.006.

[12] Yale Database A, Retrieved on January 10, 2011, from http://cvc.yale.edu/projects/yalefaces/yalefaces.html. 
